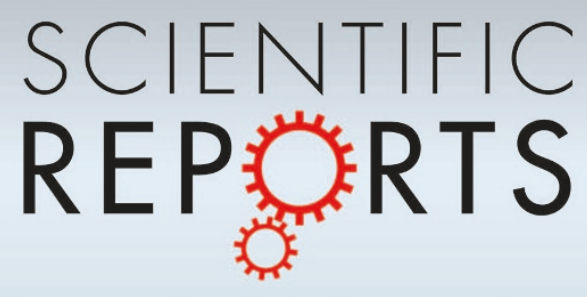

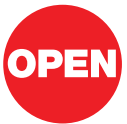

SUBJECT AREAS:

STATISTICS

ECOLOGY

THEORY

STATISTICAL PHYSICS, THERMODYNAMICS AND NONLINEAR DYNAMICS

Received

31 August 2011

Accepted

9 September 2011

Published

28 September 2011

Correspondence and requests for materials should be addressed to

B.M.W. (bmweon@ hotmail.com) or J.H.J. (jhje@postech.ac.kr)

\section{Plasticity and rectangularity in survival} curves

\author{
Byung Mook Weon' \& Jung Ho Je'
}

'X-ray Imaging Center, Department of Materials Science and Engineering, Pohang University of Science and Technology, San 31, Hyoja-dong, Pohang, 790-784, Korea.

Living systems inevitably undergo a progressive deterioration of physiological function with age and an increase of vulnerability to disease and death. To maintain health and survival, living systems should optimize survival strategies with adaptive interactions among molecules, cells, organs, individuals, and environments, which arises plasticity in survival curves of living systems. In general, survival dynamics in a population is mathematically depicted by a survival rate, which monotonically changes from 1 to 0 with age. It would be then useful to find an adequate function to describe complicated survival dynamics. Here we describe a flexible survival function, derived from the stretched exponential function by adopting an age-dependent shaping exponent. We note that the exponent is associated with the fractal-like scaling in cumulative mortality rate. The survival function well depicts general features in survival curves; healthy populations exhibit plasticity and evolve towards rectangular-like survival curves, as examples in humans or laboratory animals.

iving systems are known as open self-organizing complex adaptive systems ${ }^{1}$ and must often cope with hostile environmental conditions for health and survival ${ }^{2}$. To maintain their living (far-from-equilibrium) state, living systems should exchange matter, energy, and information from their surroundings and further adapt themselves to genetic and environmental fluctuation $\mathrm{s}^{1,2}$. To perform a variety of activities for health and survival, living systems require adaptive interactions among molecules, cells, organs, individuals, and environments or regulatory mechanisms ${ }^{3}$. A hallmark of living systems is their extraordinary complexity in physiological processes $^{3}$. Healthy living systems could optimize their survival strategies through adaptive interactions of multiple control mechanisms that enable each individual to adapt to the exigencies and unpredictable changes of everyday life $^{3}$. The formulation of survival (or mortality) curves is essential for the quantification of survival dynamics to scientists, such as demographers, biologists, and gerontologists. A single survival curve reflects a variance in survival probability (equally, survival function and survival rate) as a function of age. Despite recent advances in population biology $\mathrm{y}^{4-6}$, general features of biological survival dynamics remain elusive because of plasticity in survival curves of living systems.

Many mathematical models for survival curves have been proposed (see recent reviews ${ }^{7,8}$ ). One of the fundamental mortality laws is the Gompertz law', in which the mortality rate increases roughly exponentially with increasing age at senescence. However, it seems to be obvious that the human mortality rate does not increase according to the Gompertz law at very old ages ${ }^{8,10}$ and the deviation from the Gompertz law remains a great puzzle to demographers, biologists, and gerontologists. Many other mathematical models such as the Weibull, the Heligman-Pollard, the Kannisto, the quadratic, and the logistic models yet provide poor fit to the empirical mortality patterns at very old ages ${ }^{8}$. There are still needs for an appropriate mathematics for survival (or mortality) curves with simplicity, efficiency, and flexibility ${ }^{11-14}$. In previous works ${ }^{15-17}$, we put forward a simple survival function, which is derived from the stretched exponential function ${ }^{18-21}$. In this study, we address that this function enables us to describe general features of survival curves in healthy living systems.

The stretched exponential function is widely used to describe complex dynamics in physics and biology ${ }^{18-21}$. In physics, relaxation is an aging process in which a system gradually changes from a far-from-equilibrium (living) to an equilibrium (dead) state. Structural relaxation of a glassy state towards a metastable equilibrium amorphous state is often referred as "physical aging", which generally exhibits nonexponential relaxation ${ }^{20,21}$. The temporal behavior of the response function s(u) can be described by the stretched exponential or the Kohlrausch-WilliamsWatts $(\mathrm{KWW})$ function ${ }^{22-24}$ (often called the Weibull function ${ }^{25}$ ). This function has a general form of $\mathrm{s}(\mathrm{u})=$ $\exp \left(-\mathrm{u}^{\beta}\right)(\beta>0)$, where $\mathrm{s}(\mathrm{u})$ is the measurable quantity decreasing with age $\mathrm{u}(=\mathrm{x} / \alpha)$. Here real age $(\mathrm{x})$ can be rescaled with a characteristic life $(\alpha)$ taken at $s(\alpha)=\exp (-1) \approx 0.3679^{15}$. The KWW function appears in many complex systems from soft matter systems, such as glass-forming liquids and amorphous solids ${ }^{26,27}$, to 
astrophysical objects ${ }^{28}$. The KWW function is typically classified as the "stretched" exponential for $0<\beta<1$, the "compressed" exponential for $\beta>1$, and the "simple" exponential for $\beta=1$. The nonexponential nature $(\beta \neq 1)$ is known to result from the "dynamic heterogeneity" or the "fractal time" of the system ${ }^{29}$. In biology, the typical survival curves, $s(\mathrm{u})$, for humans or animals fall into three main types, usually known as Type-I, -II, and -III curves ${ }^{30}$. Type-I survival curves slightly change at early and middle ages and then suddenly decline at late ages, as seen for long-lived humans. Type-II curves almost linearly decease with age, as seen for shortlived birds. Type-III curves quickly decrease at early ages, as seen for most plants. Interestingly, Type-I survival curves resemble the compressed exponential curves $(\beta>1)$. Type-II curves are similar to the standard exponential curves $(\beta=1)$. Type-III curves correspond to the stretched exponential curves $(0<\beta<1)$. In this study, we identify the age dependency of $\beta$ in biological systems.

\section{Results}

Plasticity in survival curves. In our previous works, we showed that complicated survival curves are well described by modifying the KWW function with an "age-dependent" shaping exponent $\beta(u)$, as follows ${ }^{15-17}$ :

$$
s(u)=\exp \left(-u^{\beta(u)}\right)
$$

In this work, we argue that the age dependence of $\beta(\mathrm{u})$ can be understood in terms of fractal time $u^{\beta(u)}$ replacing time $u^{29}$. Conceptually, fractal time describes highly intermittent self-similar temporal behavior that does not possess a characteristic time scale ${ }^{29,31}$. The cumulative mortality (hazard) function, $\mathrm{h}(\mathrm{u})=-\ln (\mathrm{s})=\mathrm{u}^{\beta(\mathrm{u})}$, (defined as the negative logarithmic survival function, equally the fractal time in our case) shows a typical fractal-like scaling as $\mathrm{h}(\mathrm{bu})=\mathrm{b}^{\beta(\mathrm{u})} \mathrm{h}(\mathrm{u})$ for an arbitrary constant $\mathrm{b}$. By rescaling $\mathrm{u}$, instead of $\mathrm{x}$, we are able to discover a universal, species-independent, scaleinvariant survival law from different species. Interestingly, we note that $\beta(\mathrm{u})$ may vary with time in physical systems; for instance, luminescence decays ${ }^{15}$ or carrier hopping dynamics ${ }^{32}$. Most importantly, we emphasize that the shaping exponent $\beta(\mathrm{u})$ is a dynamic variable with age for biological systems. The age dependency of $\beta(u)$ would shed light on how living systems evolve towards their "slowest aging rates" for healthy survival, which would be a key feature of survival dynamics in living systems.

We describe the basic assumptions of our mathematical approach. We assume three points as follows. (1) Living systems keep trying to survive by adapting themselves to internal or external conditions, including genetic (biological) and non-genetic (environmental) conditions. (2) Survival strategies in living systems would be optimized for better survival. Survival strategies would be consequences of all functional interactions among genes, molecules, cells, organs, and individuals within living systems or with environments. Finally, (3) a survival function depicts survival dynamics of an individual or a population. In fact, historic trends in human survival curves show a gradual evolution toward healthy aging. We are able to describe those trends in human survival curves with the stretched exponential function $\mathrm{s}(\mathrm{u})=\exp \left(-\mathrm{u}^{\beta(\mathrm{u})}\right)$ by adjusting the age-dependent $\beta(\mathrm{u})$. Biological differences from species (scaling effect) may be cancelled by rescaling age (the $\mathrm{x}$ axis) as illustrated in Fig. 1. Using the agedependent, shaping exponent, $\beta(\mathrm{u})$, we show how survival curves evolve towards the rectangular-like curves.

Rectangularity in survival curves. We think of the concept of "healthy aging", which may be indeed an ultimately adaptive feature in survival curves of living systems. As a typical example, we consider survival curves for humans. From life table data of human populations in developed countries, we find that survival probability evolves over time (remarkably, for last several decades), universally towards a rectangular shape, as many scientists observed ${ }^{33-35}$. Such a gradual
$0 \leq S(u)=\exp \left(-u^{\beta(u)}\right) \leq 1$

Scaling effect $\rightarrow$ determined by a

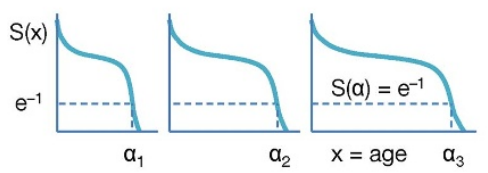

Scale invariance by rescaling $u=x / \alpha$

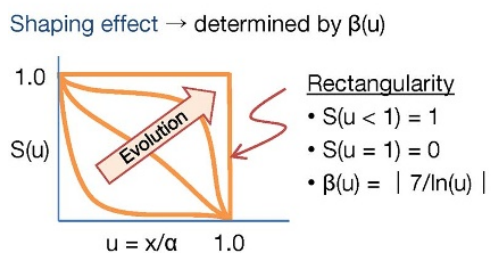

Figure $1 \mid$ Basic model. Survival curves are well described with the stretched exponential survival function $s(u)=\exp \left(-u^{\beta(u)}\right)$ (see the details in the text) by adjusting the age-dependent, shaping exponent, $\beta(u)$. Biological differences from species (scaling effect) can be cancelled by rescaling age $\mathrm{u}=\mathrm{x} / \alpha$, where the characteristic life $(\alpha)$ is graphically taken at $\mathrm{s}(\mathrm{x}=\alpha)=\exp (-1)$. Using the age-dependent, shaping exponent, $\beta(\mathrm{u})$, we are able to describe how survival curves evolve towards the rectangular-like curves.

evolution in human survival curves, as called the "rectangularization" ${ }^{33}$, results from the fact that survival curves become more rectangular and deaths are compressed to higher ages. How can we describe the evolution in human survival curves? Presumably the healthy aging can be characterized as the "slowest aging rates", equivalently indicating that the mortality rates are minimized at each age. Here we suggest a simple mathematical approach, which would be useful to test the validity of the rectangularization hypothesis.

By modeling $\mathrm{s}(\mathrm{u})$ as a modified stretched exponential function with an age-dependent shaping exponent, we are able to evaluate the slowest aging rates ${ }^{16,17}$. Generally speaking, survival function $\mathrm{s}(\mathrm{u})$ decreases monotonically from 1 to 0 with age; that is, $-\mathrm{ds}(\mathrm{u}) /$ $\mathrm{du}>0$. Let $-\mathrm{ds}(\mathrm{u}) / \mathrm{du} \rightarrow 0$ denote the slowest aging rates, equivalently implying the largest survival rates $(\approx 1$ at $\mathrm{u}<1)$. We find that $-\mathrm{ds}(\mathrm{u}) / \mathrm{du} \rightarrow 0$ can be achieved only for a positive slope of $\beta(\mathrm{u})$ (i.e., $d \beta / d u>\varepsilon)$ early in life $(u<1)$ or for a negative slope of $\beta(u)$ (i.e., $d \beta / d u>\varepsilon)$ at late life $(u>1)$, since the quantity $\varepsilon=-\beta / u \ln (u)$ is positive at $\mathrm{u}<1$ and negative at $\mathrm{u}>1^{16,17}$. Using the age dependence of $\beta(u)$, we are able to describe the slowest aging rates in living systems.

Here we derive a simple mathematics to determine the slowest aging rates. By solving the equation $d \beta / d u=-\beta / u \ln (u)$ to satisfy "-ds $(\mathrm{u}) / \mathrm{du}=0$ ", we obtain an ideal curve of $\beta(\mathrm{u})$ that holds if and only if the derivative of the survival probability approaches zero, implying the "slowest aging rates". This condition reflects the rectangular survival curve, where $\mathrm{s}(\mathrm{u})$ becomes completely rectangular in which all individuals are alive with $\mathrm{s}(\mathrm{u}) \approx 1$ to its characteristic life $(\mathrm{u}=1)$ and suddenly die with $\mathrm{s}(\mathrm{u}) \approx 0$ after $\mathrm{u}>1$. Therefore the slowest aging rates of living systems can be simply described as:

$$
\beta(u)=\frac{c}{|\ln (u)|}
$$

Here, $\mathrm{c}$ is a universal constant (numerically $\mathrm{c} \geq 7$ for $\mathrm{s}>0.999089$ at $\mathrm{u}<1$ ). Most importantly, the rectangular survival curve can be precisely approximated with $\beta(\mathrm{u})$ for $\mathrm{c}=7$. This gives a criterion for rectangularity of survival curves: when living systems evolve to achieve the maximum survival probability, $\beta(\mathrm{u})$ should vary towards the ideal curve, $\beta(u)=7|\ln (u)|^{-1}$. Therefore, $\beta(u)$ is a good measure to determine the appearance of the rectangularity in survival curves. 
Application to human demographic data. We test the validity of our mathematical approach in real systems. For typical human survival curves, the age-related trajectory of $\beta(\mathrm{u})$ increases with age towards the ideal curve of $\beta(\mathrm{u})$ at early ages $(\mathrm{u}<1)$, as fitted by a linear form of $\beta_{0}+\beta_{1} u$, and in turn decreases with age at late ages $(\mathrm{u}>1)$, as fitted by a quadratic form ${ }^{16,17}$ as $\beta_{0}+\beta_{1} u+\beta_{2} u^{2}$. For instance, from Swedish female periodic life table in 2000 , we roughly estimate $\beta(\mathrm{u})$ with $\beta_{0} \approx 1$ and $\beta_{1} \approx 9$ at $\mathrm{u}<1$ and $\beta_{0} \approx-20, \beta_{1} \approx 60$, and $\beta_{2} \approx-30$ at $\mathrm{u}>1$, as depicted as circles in Fig. 2. As illustrated in the inset, the survival curve obtained from the estimated $\beta(\mathrm{u})$ (circles) quite well agrees with the actual survival curve of Swedish females in 2000 (dashed line). Remarkably, the estimated and the measured survival curves are very close to the rectangular-like survival curve (solid line).

The evolution towards the slowest aging rates makes survival curves to have rectangularity. We test this feature for humans with the reliable demographic data taken from the Human Mortality Database (http://www.mortality.org). The survival curves are analyzed by computing the age-dependent shaping exponents $\beta(\mathrm{u})=$ $\ln (-\ln (\mathrm{s})) / \ln (\mathrm{u})$ with rescaled age $(\mathrm{u}=\mathrm{x} / \alpha)$ from the general expression of survival probability $s=\exp \left(-\mathrm{u}^{\beta(\mathrm{u})}\right)$, where the characteristic life $(\alpha)$ is graphically taken at $s(\alpha)=\exp (-1)$ for each survival curve. We find that Swedish females' survival curves during last two centuries from 1800 to 2000 corroborate that the age-related trajectory of $\beta(u)$ evolves in a fashion predicted in theory (i.e., towards the ideal curve, $\beta(\mathrm{u})=7|\ln (\mathrm{u})|^{-1}$, solid line), as illustrated in Fig. 3 . The agedependent evolution of $\beta(\mathrm{u})$ provides conclusive evidence that human survival curves evolve towards the slowest aging rates, which supports the validity of the rectangularization hypothesis ${ }^{33}$.

Let us consider mortality rate (sometimes called hazard function or force of mortality), defined as a negative derivative of logarithmic survival rate. The age-related evolution of $\beta(\mathrm{u})$ has clues to interpret the age-related evolution of mortality rate. The mortality rate $\mu(\mathrm{u})=-\mathrm{d} \ln (\mathrm{s}) / \mathrm{du}$ in our model is described with respect to the age-dependent $\beta(\mathrm{u})$ as:

$$
\mu(u)=\left(\frac{u^{\beta(u)}}{\alpha}\right)\left[\frac{\beta(u)}{u}+\ln (u) \frac{d \beta(u)}{d u}\right]
$$

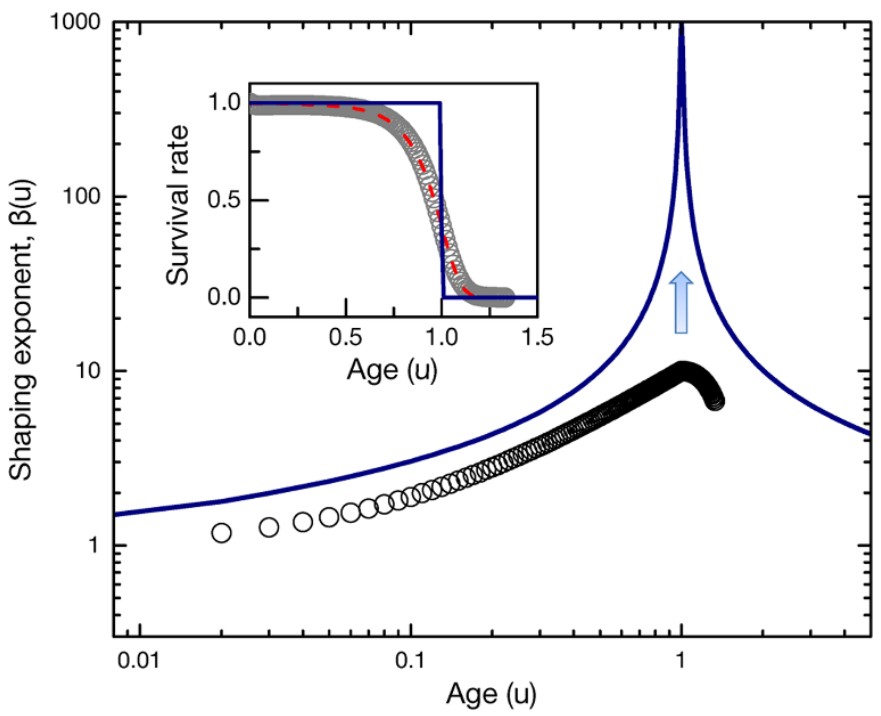

Figure $2 \mid$ Healthy survival curves. The shaping exponent, $\beta(\mathrm{u})$, approaches to the ideal curve, $\beta(\mathrm{u})=7|\ln (\mathrm{u})|^{-1}$ (solid line), as the survival probability, $\mathrm{s}(\mathrm{u})$, approaches to the rectangular shape (solid line, inset). This tendency, which stands for healthy aging, is obvious in a typical trajectory of $\beta(\mathrm{u})$ for humans (circles, see the fitting parameters in the text). The survival curve estimated from the typical $\beta(\mathrm{u})$ (circles, inset) agrees well with the real survival curve for Swedish females in 2000 (dashed line, inset).

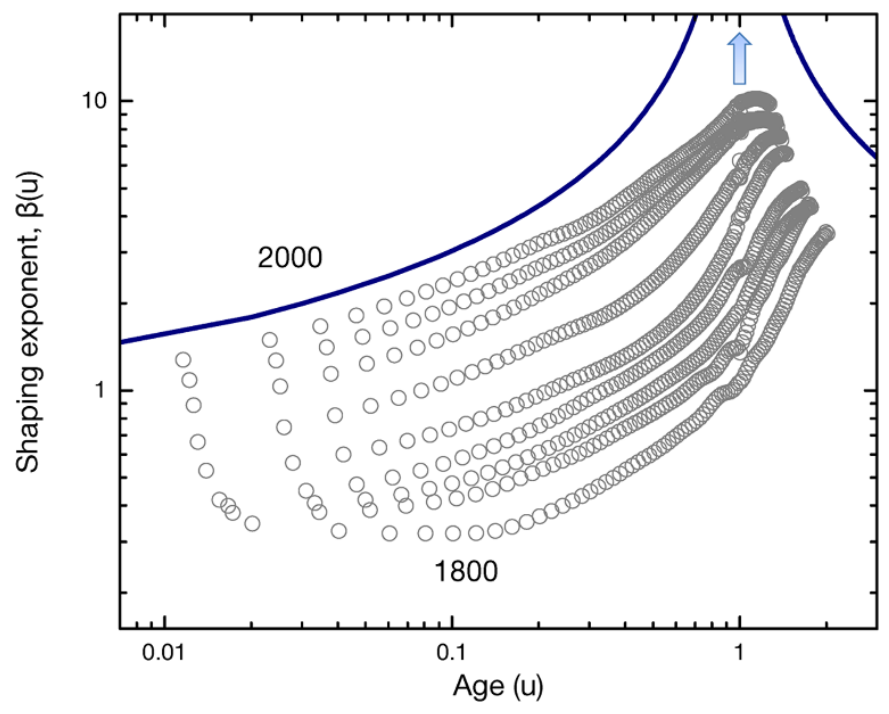

Figure 3 Evolution of shaping exponents for humans. The historic evolution in survival curves for Swedish females shows that $\beta(\mathrm{u})$ dynamically evolves towards the slowest aging rates $\left(\beta(\mathrm{u})=7|\ln (\mathrm{u})|^{-1}\right.$, solid line), where $\mathrm{s}(\mathrm{u})$ becomes rectangular for last two centuries (1800-2000 years).

Here, the mortality rate can be calculated from $\alpha$ and $\beta(\mathrm{u})$ for a single survival curve. For ideal survival conditions, $\beta(\mathrm{u})$ tends to evolve towards the ideal curve of $\beta(u)$ and the mortality rate should become zero for all ages. For real survival conditions (for humans or animals), as the survival curve approaches to a rectangular shape, $\beta(\mathrm{u})$ tends to linearly increase with age at early ages $(\mathrm{u}<1)$, which corresponds to a linear increase of $\ln (\mu)$. The linearity in $\ln (\mu)$ at middle ages ( $\mathrm{u}=0.5-1.0$, equivalently $30-80$ years for humans) is similar to the Gompertz mortality law $w^{17}$. At late ages $(u>1), \beta(u)$ shows a quadratic change with age, which corresponds to a quadratic change of $\ln (\mu)$. The quadratic decline of $\ln (\mu)$ is practically valid for the oldest-age mortality patterns ${ }^{34}$. We find a general scaling relation between $\beta(\mathrm{u})$ and $\ln (\mu)$; that is, $\beta(\mathrm{u})$ is approximately proportional to $\ln (\mu)$, which is valid as shown in Figs. 3 and 4.

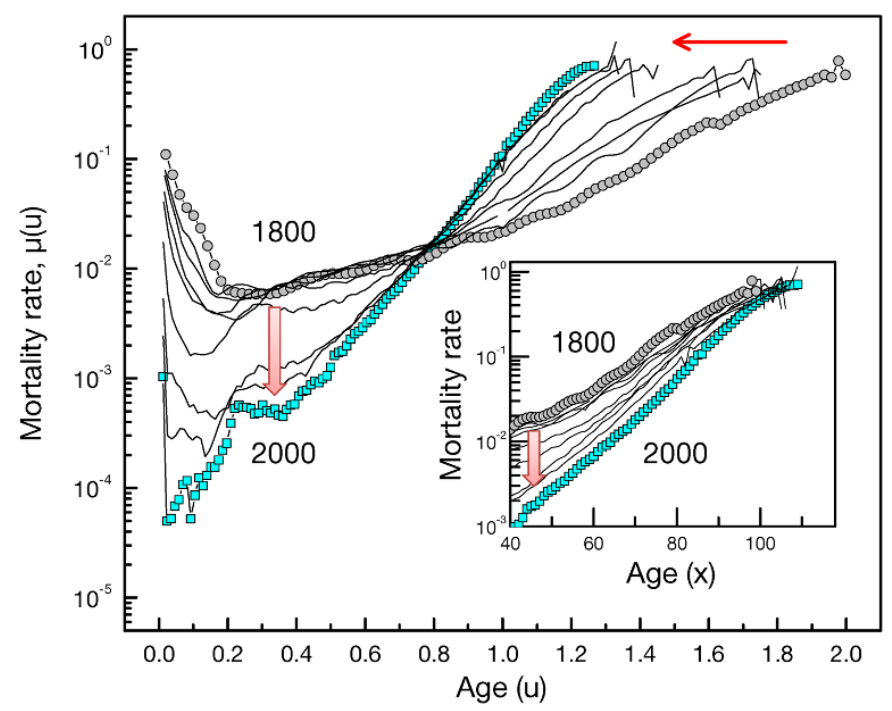

Figure 4 | Evolution of mortality rates for humans. The mortality curves for Swedish females for the last two centuries demonstrate that human mortality curves evolve towards the slowest aging rates. Interestingly, at late ages the mortality seems to be fixed (parallel shift, $\leftarrow$ ) over time, while at early ages it significantly falls down (vertical shift, $\downarrow$ ). Here, $\mu(\mathrm{u})$ linearly increases with reduced age $(\mathrm{u})$ at middle ages, showing the Gompertz law, and converges with real age $(\mathrm{x}=\alpha \mathrm{u}$ [years] with its characteristic life $\alpha$ ) at old ages (inset). 
In principle, the mortality rate is predicted to reach zero for $\mathrm{u}<1$, if the survival rate approaches to the rectangular curve. This feature can be identified by evaluating the age-related evolution of $\beta(u)$, which gets close to the "ideal" curve of $\beta(u)=7|\ln (u)|^{-1}$. In practice, it would be relatively easy to diminish the mortality rate to a very low level at young ages through health care and welfare but not at old ages by age-associated progressive aging. We find that the mortality rates of the oldest seem to be fixed at $\sim 1$ (parallel shift, $\leftarrow$ ) over time for humans, while the infant mortality rates plastically fall downward (vertical shift, $\downarrow$ ), as shown in Fig. 4. Such parallel and vertical shifts of the mortality rates imply that death is being delayed because people are reaching old age in better health ${ }^{36}$. We also find a convergence of $\mu(\mathrm{u})$ at old ages as depicted in Fig. 4. This convergence would universally exist in living systems ${ }^{37}$ if death rates become relatively similar at old ages for a single species.

Universality in non-human systems. We further investigate the agerelated evolution of $\beta(\mathrm{u})$ in non-human systems (Fig. 5). The reliable life tables were taken for wild-type flies (Drosophila melanogaster, $\alpha=46.38$ days) ${ }^{38}$, worms (Blatta orientalis, $\alpha=45.39$ days) ${ }^{38}$, automobiles $(\alpha=8.08 \mathrm{yrs})^{38}$, and tyrannosaurs (Albertosaurus sarcophagus, $\alpha=17.45 \mathrm{yrs})^{39}$. The data were arbitrary selected for analysis. Indeed, $\beta(\mathrm{u})$ dynamically evolves with age for living systems, which is remarkably shown in flies, while $\beta(\mathrm{u})$ is relatively constant $(\beta \approx 2)$ for automobiles as non-living systems. Most importantly, the age-related trajectory of $\beta(\mathrm{u})$ for flies is very similar to that for humans, approaching to the ideal curve, $\beta(\mathrm{u})=7|\ln (\mathrm{u})|^{-1}$. Additionally, the mortality rate of flies at $\mathrm{u}=0.5-1.0$ linearly increases with age, showing the validity of the Gompertz law, and also comes to a saturation at $\mathrm{u}>1$, showing the singularity of the mortality plateau. These results suggest that the common features of mortality curves such as the Gompertz law and the mortality plateau might spontaneously emerge from the age-dependent shaping exponents that dynamically evolve towards the slowest aging rates of living systems.

For both cases of humans and flies, $\beta(\mathrm{u})$ gradually evolves with age, approaching to the ideal curve, $\beta(\mathrm{u})=7|\ln (\mathrm{u})|^{-1}$, as predicted in theory. The universality of mortality patterns is well confirmed for

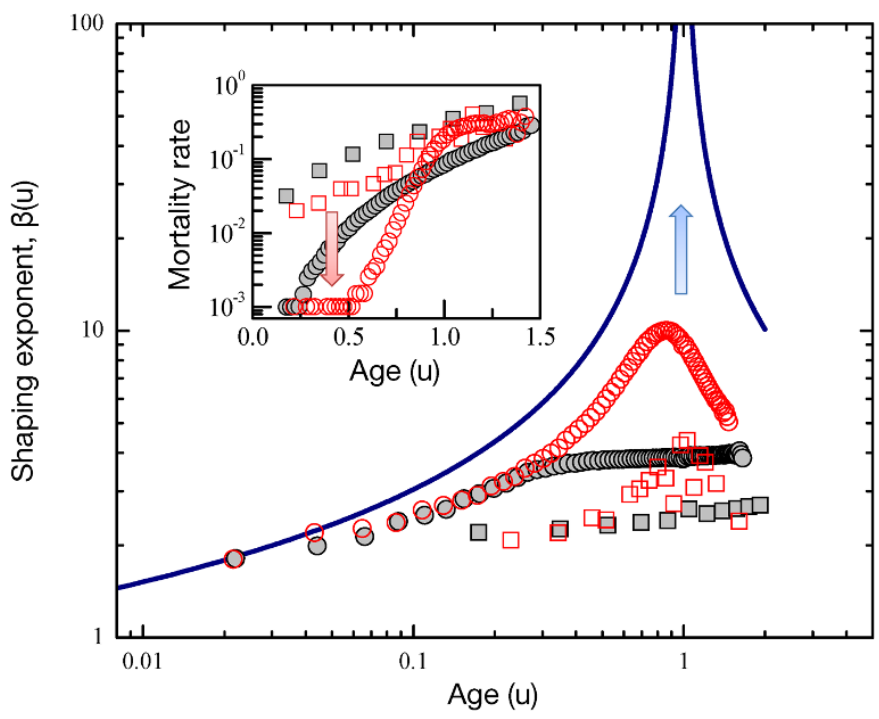

Figure 5 Evolutions of shaping exponents and mortality rates for non-human systems. The feature of the slowest aging rates (solid line, $\left.\beta(\mathrm{u})=7|\ln (\mathrm{u})|^{-1}\right)$ emerges for wild-type flies (Drosophila melanogaster, open circles), worms (Blatta orientalis, closed circles), and tyrannosaurs (Albertosaurus sarcophagus, open squares). In contrast, automobiles (closed squares) show a constant value of $\beta$ and a high value of $\mu$, indicating typical non-living systems. insects, worms, and yeasts, as well as humans in many studies ${ }^{5,34}$. It is important to note that the evolution towards the maximum survival probability is scale-invariant regardless of biological species. This suggests that the evolution towards the slowest aging rates would be a species-independent, scale-invariant, universal aspect in survival dynamics of living systems.

\section{Discussion}

Why does the dynamic nature in the shaping exponent $\beta(\mathrm{u})$ emerge in living systems? Living systems are ubiquitous in our daily life: specifically, if processes underlying life are explained as a tendency towards maximum entropy production, systems such as galaxies and hurricanes might be described as living systems ${ }^{40}$. Living systems should be capable of altering strategies and of adapting to internal and external changes. Healthy physiological functions require an integration of complex networks of control systems and feedback loops that operate on multiple scales in space and time ${ }^{3}$. These fractal-like physiological processes enable an organism to adapt to the exigencies of everyday life. In general, the complexity of living processes should be evolvable with environmental conditions. Adaptive interactions of each individual occur in a cooperative manner with its neighbors, resulting in dynamic self-organization and influencing flexible survival strategies between cooperation and competition. Since living systems would evolve to optimize their capabilities and strategies for survival, the slowest aging rates would spontaneously emerge. Presumably, living processes would not follow simple physical relaxation processes, where the shaping exponent is invariant with age. To illustrate evolvable survival curves of living systems, we adopt an age-related shaping exponent, $\beta(\mathrm{u})$, which provides a simple criterion for the slowest aging rates. On this basis, dynamic change in shaping exponent may be attributed to "complexity evolution" in living systems. On the other hand, complexity evolution would be able to give hints about how and why vitality declines with age $^{41}$. Consequently, the appearance of the age-dependent $\beta(u)$ in living systems is due to the evolution towards the slowest aging rates and fundamentally attributed to the complexity evolution (agerelated evolution in complexity) for living systems.

Finally, we note that a mathematics-based survival model has a limitation yet. A more direct link should be established between the model and the current understanding of aging mechanisms at a genetic/molecular level ${ }^{42}$. Such linkage is beyond the scope of this study. The aging dynamics cannot be always straightforwardly explained as a loss of complexity ${ }^{43}$. To better understand the complexity evolution in biological aging dynamics, network theory ${ }^{44,45}$ or systems biology ${ }^{46}$ would be essential. We believe that cooperation and competition between components would complicatedly contribute to the optimum survival strategy and the aging dynamics ranging from generic to systematic levels. Further study is required to develop the mathematics-biology linkage in survival dynamics. On the other hand, our model well describes the rectangularization trends in the survival curves, as simply characterized as the $\beta(\mathrm{u})$ curve that approaches to $7|\ln (\mathrm{u})|^{-1}$. These trends were traditionally explained by the Gompertz-Makeham law of mortality with the Makeham parameter changes ${ }^{47,48}$. Our mathematical interpretation offers a quite quantitative criterion to confirm the rectangularization tendency, compared with the classical demographic explanation of the rectangularization.

In summary, we put forward a simple mathematics to describe plasticity and rectangularity in survival curves of living systems. Our description is useful to illustrate how living systems evolve towards the rectangular survival curves through the slowest aging rates. Such an elegant evolution is testable with a simple mathematical criterion derived from the age dependence of the shaping exponent in the stretched exponential survival function. The age-dependent shaping exponent is a useful indicator of plasticity in survival curves. The feature of the slowest aging rates emerges in survival curves for 
humans and laboratory flies. Our finding may give more useful insights into basic aspects of survival dynamics, "plasticity and rectangularity", in living systems by offering a mathematical description of healthy survival dynamics.

\section{Methods}

The survival curves were analyzed by computing the age-dependent shaping exponents $\beta(\mathrm{u})=\ln (-\ln (\mathrm{s})) / \ln (\mathrm{u})$ with rescaled age $(\mathrm{u}=\mathrm{x} / \alpha)$ from the general expression of survival probability $s=\exp \left(-\mathrm{u}^{\beta(\mathrm{u})}\right)$, where the characteristic life $(\alpha)$ is graphically taken at $s(\alpha)=\exp (-1)$ from each survival curve $e^{16,17}$. We used the reliable demographic data for humans, Swedish females' survival curves during last two centuries from 1800 to 2000, taken from the Human Mortality Database (http://www.mortality.org). The reliable life tables for animals were taken for wildtype flies (Drosophila melanogaster, $\alpha=46.38$ days) ${ }^{38}$, worms (Blatta orientalis, $\alpha=45.39$ days $)^{38}$, automobiles $(\alpha=8.08 \mathrm{yrs})^{38}$, and tyrannosaurs (Albertosaurus sarcophagus, $\alpha=17.45 \mathrm{yrs})^{39}$.

1. Coffey, D. S. Self-organization, complexity and chaos: the new biology for medicine. Nat. Med. 4, 882-885 (1998).

2. Ben-Jacob, E., Cohen, I. \& Levine, H. The cooperative self-organization of microorganisms. Adv. Phys. 49, 395-554 (2000).

3. Lipsitz, L. A. \& Goldberger, A. L. Loss of complexity and aging. J. Am. Med. Assoc. 267, 1806-1809 (1992); Goldberger, A. L., et al. Fractal dynamics in physiology: alterations with disease and aging. Proc. Natl. Acad. Sci. USA 99, 2466-2472 (2002)

4. Coe, J. B., Mao, Y. \& Cates, M. E. Solvable senescence model showing a mortality plateau. Phys. Rev. Lett. 89, 288103 (2002).

5. Azbel, M. Y. Empirical laws of survival and evolution: Their universality and implications. Proc. Natl. Acad. Sci. USA 96, 15368-15373 (2002); Azbel, M. Y. Law of universal mortality. Phys. Rev. E 66, 016107 (2002).

6. Partridge, L. \& Mangel, M. Messages from mortality: the evolution of death rates in the old. Trends Ecol. Evol. 14, 438-442 (1999).

7. Wachter, K. W. \& Finch, C. (eds) Between Zeus and the Salmon: the biodemography of longevity. (National Academic Press: Washington DC, 1997).

8. Thatcher, A. R., Kannisto, V. \& Vaupel, J. W. The force of mortality at ages 80-120. (Odense monographs on population aging 5. Odense University Press: Odense, 1998).

9. Gompertz, B. On the nature of the function expressive of the law of human mortality. Philos. Trans. R. Soc. Lond. A 115, 513-580 (1825).

10. Robine, J. M. \& Vaupel, J. W. Emergence of supercentenarians in low mortality countries. N. Am. Actuarial J. 6, 54-63 (2002)

11. Klawansky, S. \& Fox, M. S. A joint hazard and time scaling model to compare survival curves. Proc. Natl. Acad. Sci. USA 93, 8183-8188 (1996).

12. Pletcher, S. D. Model fitting and hypothesis testing for age- specific mortality data. J. Evol. Biol. 12, 430-439 (1999).

13. Frank, S. A. A multistage theory of age-specific acceleration in human mortality. BMC Biol. 2, 16 (2004).

14. Ahmed, F. E., Vos, P. W. \& Holbert, D. Modeling survival in colon cancer: a methodological review. Molecular Cancer 6, 15 (2007).

15. Weon, B. M., Lee, J. L. \& Je, J. H. A unified decay formula for luminescence decays. J. Appl. Phys. 98, 096101 (2005); Weon, B. M., Je, J. H. \& Lee, J. L. Lifetime dispersion in a single quantum dot. Appl. Phys. A 89, 1029-1031 (2007).

16. Weon, B. M. \& Je, J. H. Theoretical estimation of maximum human lifespan. Biogerontology 10, 65-71 (2009).

17. Weon, B. M. \& Je, J. H. Predicting human lifespan limits. Natural Science 2, $984-$ 989 (2010).

18. Vlad, M. O., Schönfisch, B. \& Mackey, M. C. Fluctuation-dissipation relations and universal behavior for relaxation processes in systems with static disorder and in the theory of mortality. Phys. Rev. E 53, 4703-4710 (1996).

19. Metzler, R., Klafter, J. \& Jortner, J. Hierarchies and logarithmic oscillations in the temporal relaxation patterns of proteins and other complex systems. Proc. Natl. Acad. Sci. USA 96, 11085-11089 (1999).

20. Debenedetti, P. G. \& Stillinger, F. H. Supercooled liquids and the glass transition. Nature 410, 259-267 (2001)

21. Richert, R. Physical aging and heterogeneous dynamics. Phys. Rev. Lett. 104, $085702(2010)$

22. Kohlrausch, R. Theorie des elektrischen rückstandes in der leidener flasche. Pogg. Ann. Phys. Chem. 91, 179-214 (1854).

23. Williams, G. \& Watts, D. C. Non-symmetrical dielectric relaxation behavior arising from a simple empirical decay function. Trans. Faraday Soc. 66, 80-85 (1970).
24. Cardona, M., Chamberlin, R. V. \& Marx, W. The history of the stretched exponential function. Ann. Phys. (Leipzig) 16, 842-845 (2007).

25. Weibull, W. A. A statistical distribution function of wide applicability. J. Appl. Mech. 18, 293-297 (1951).

26. Tang, C. \& Bak, P. Critical exponents and scaling relations for self-organized critical phenomena. Phys. Rev. Lett. 60, 2347-2350 (1988).

27. Berberan-Santos, M. N., Bodunov, E. N. \& Valeur, B. Mathematical functions for the analysis of luminescence decays with underlying distributions 1 . Kohlrausch decay function (stretched exponential). Chem. Phys. 315, 171-182 (2005).

28. Dobrovolskis, A. R., Alvarellos, J. L. \& Lissauer, J. J. Lifetimes of small bodies in planetocentric (or heliocentric) orbits. Icarus 188, 481-505 (2007).

29. Shlesinger, M. F. Fractal time in condensed matter. Annu. Rev. Phys. Chem. 39, 269-290 (1988)

30. Demetrius, L. Adaptative value, entropy and survivorship curves. Nature $\mathbf{2 7 5}$, 213-214 (1988)

31. Plonka, A. Fractal-time dynamics of elementary reactions in condensed media. Kinetics and Catalysis 37, 661-665 (1996)

32. Scher, H. \& Montroll, E. W. Anomalous transit-time dispersion in amorphous solids. Phys. Rev. B 12, 2455-2477 (1975)

33. Fries, J. F. Aging, natural death, and the compression of morbidity. N. Engl. J. Med. 303, 130-136 (1980).

34. Vaupel, J. W., et al. Biodemographic trajectories of longevity. Science 280, 855-860 (1999).

35. Olshansky, S. J., et al. A potential decline in life expectancy in the United States in the 21st century. N. Engl. J. Med. 352, 1138-1145 (1999).

36. Vaupel, J. A. Biodemography of human aging. Nature 464, 536-542 (2010).

37. Gavrilov, L. A. \& Gavrilova, N. S. The reliability theory of aging and longevity. J. Theor. Biol. 213, 527-545 (2001).

38. Pearl, R. \& Miner, J. R. Experimental studies on the duration of life. XIV. The comparative mortality of certain lower organisms. Q. Rev. Biol. 10, 60-79 (1935).

39. Erickson, G. M., Currie, P. J., Inouye, B. D. \& Winn, A. A. Tyrannosaur life tables: an example of nonavian dinosaur population biology. Science 313, 213-217 (2006).

40. Whitfield, J. Survival of the likeliest? PLoS Biol. 5, e142 (2007).

41. Moorad, J. A. \& Promislow, D. E. L. A theory of age-dependent mutation and senescence. Genetics 179, 2061-2073 (2008).

42. Partridge, L. \& Gems, D. Mechanisms of aging: public or private? Nat. Rev. Gen. 3 , 165-175 (2002).

43. Shiogai, Y., Stefanovska, A. \& McClintock, P. V. E. Nonlinear dynamics of cardiovascular ageing. Phys. Rep. 488, 51-110 (2010).

44. Wang, S., Szalay, M. S., Zhang, C. \& Csermely, P. Learning and innovative elements of strategy adoption rules expand cooperative network topologies. PLoS ONE 3, e1917 (2008)

45. Kiss, H. J. M., et al. Ageing as a price of cooperation and complexity. BioEssays 31, 651-664 (2009).

46. Kirkwood, T. B. L. Systems biology of ageing and longevity. Phil. Trans. R. Soc. B 366, 64-70(2011)

47. Gavrilov, L. A., Gavrilova, N. S. \& Nosov, V. N. Human life span stopped increasing: why? Gerontology 29, 176-180 (1983).

48. Gavrilov, L. A. \& Gavrilova, N. S. The Biology of Life Span: A Quantitative Approach. (Harwood Academic Publisher: New York, 1991).

\section{Acknowledgements}

This research was supported by the Creative Research Initiatives (Functional X-ray Imaging) of the Ministry of Education, Science, and Technology of Korea (MEST) and the National Research Foundation of Korea (NRF).

\section{Author contributions}

B.M.W. conceived of the research, complied and analyzed the data, and was the primary writer; J.H.J. contributed to discussion and the final manuscript.

\section{Additional information}

Competing financial interests: The authors declare no competing financial interests.

License: This work is licensed under a Creative Commons

Attribution-NonCommercial-ShareAlike 3.0 Unported License. To view a copy of this license, visit http://creativecommons.org/licenses/by-nc-sa/3.0/

How to cite this article: Weon, B.M. \& Je, J.H. Plasticity and rectangularity in surviva curves. Sci. Rep. 1, 104; DOI:10.1038/srep00104 (2011). 